\title{
IV. Remarks on the atomic constitution of elastic fluids
}

\section{William Charles Henry M.D. F.R.S.}

To cite this article: William Charles Henry M.D. F.R.S. (1834) IV. Remarks on the atomic constitution of elastic fluids, Philosophical Magazine Series 3, 5:25, 33-39, DOI:

10.1080/14786443408648395

To link to this article: http://dx.doi.org/10.1080/14786443408648395

册 Published online: 01 Jun 2009.

Submit your article to this journal $\lceil\pi$

Џ Article views: 3

Q View related articles $₫$ 
over the countries through which they flow; the plain of Shinar is the scene in which the history of mankind recommences when the Deluge is over. Unless we could suppose the Pentatench to have been written after the captivity, and the Jews to have begun their history with the borrowed traditions of their oppressors, we must admit that these things were subjects of belief in the family of Abraham from the time when he left his original abode in Ur of the Chaldees*.

IV. Remarks on the Atomic Constitution of Elastic Fluids. By William Charles Henry, M.D., F.R.S.†

THE following remarks, suggested by that portion of $\mathrm{Dr}$.

Prout's Bridgewater Treatise which is devoted to the most comprehensive generalizations of chemical philosophy, are proposed with considerable hesitation, from their not according with the views of that profound writer. But it must also be borne in mind, that the theory of atomic combination adopted by Dr. Prout differs itself, most materially, from that originally framed by the author of the atomic philosophy, and still held by him, as well as by the majority of British chemists. These differences, as far as they respect first principles, may be comprehended in the two following propositions:

ist, That equal volumes of all gaseous bodies contain, under the same temperature and pressure, the same number of self-repulsive molecules.

2nd, "That the self-repulsive molecule, as it exists in the gaseous form, does not represent the ultimate molecule, but is composed of many of them."

1st, The idea that the particles of all gaseous fluids are placed at the same distances from one another, and consequently that a given space contains in all the same number of molecules, seems to have occurred about the same time to MM. Ampère and Avogadro. It was published by the former, so early as the year 1814, in a letter addressed to Count Berthollet, but merely as the most probable hypothesis of the constitution of elastic matter $\neq$. It was subsequently revived by Dumas, and has been recently maintained and illustrated by his pupil M. Gaudin§. Dr. Prout had arrived at the same

- [Our correspondent Mr. Beke, in the Appendix to his recently published work, entitled Origines Biblice, has shown reasons for the belief that the Flood, though universal with respect to mankind, was merely local with respect to the globe itself; a view of the subject which, if fully substantiated, would tend to relieve it from much of the difficulty in which it is at present involved.-EDIT.]

tCommunicated by the Author. $₫$ Ann. de Chimie, tom. xc. p. 47.

$\$$ Ann. de Chimie et de Phys., tom. lii. p. 113.

Third Series. Vol. 5. No. 25. July 1834. 
conception without being aware that it had been previously entertained by others. These distinguished chemists do not, however, concur with Ampère in regarding it in the light merely of an hypothesis, but conceive that it is strictly derivable from the well-known law of Mariotte, and from the similar relations of gaseous bodies to heat. They have therefore made the first of the above propositions the basis of their peculiar views on atomic combination, and have certainly succeeded in proving that the second and more important proposition flows from the first in direct logical sequence. It is therefore necessary to examine, with peculiar care, the grounds upon which the major term is supposed to rest.

The law of Mariotte, that in all elastic fluids the volumes vary inversely as the compressing forces, will be found to warrant no inference as to the number of atoms existing in a given volume of the different gases. It is derived from the law of variation observed by the repulsive forces which actuate the molecules of elastic fluids, not from the numerical aggregation of atoms in space. Newton has demonstrated (Princ., Lib. II. Pr. xxiii.) " that particles flying from each other with forces that are reciprocally proportional to the distances of their centres, compose an elastic fluid whose density is as the compression." Now this is the law of Mariotte, which is hence independent of all elements other than repulsive forces, varying inversely as the atomic distances or diameters. Whatever be the comparative distances of the particles of two gases $A$ and $B$ under any given pressure, the same for both, there must, in conformity with the law of Newton, be an equal diminution of their bulk on equal increments of pressure. For illustration, let us suppose the atoms of $\mathbf{A}$ to be at double the distance that exists between the atoms of $B$ under the pressure of one atmosphere. Let the two gases be subjected to the pressure of an additional atmosphere. Then, since the molecular forces in both vary according to the same law, both gases will be alike reduced to half their original volume. But the number of atoms of $B$ is eight times that of $\mathbf{A}$. Hence it is manifest that the law of Mariotte has no reference whatever to the numerical relations of atoms in the different gases.

2nd, The argument founded on the equal expansion of the gases by heat, does not appear to be possessed of greater cogency. In the first place, it is consistent with the best recent experiments, as will be shown hereafter, that equal increments of absolute heat do not produce equal dilatations of volume in the different gases, and that this relation can only be correctly predicated of equal increments of temperature. Now there seems 
more reason to anticipate the existence of such a relation between the number of molecules and the absolute or specific heat, than between that number and the heat of temperature. Upon this principle, since unequal increments of absolute heat are required to effect equal expansions, we should conclude that the numbers of atoms are also unequal.

That equal increments of temperatures should affect all elastic fluids in the same degree, is a manifest consequence of the constitution of such fluids. The unequal expansibility of bodies in the solid and liquid states is to be ascribed to the interference of the attractive forces which maintain those conditions of matter, and which counteract, with energies varying in different bodies and at different distances, the repulsive agency of heat. But the molecules of elastic fluids are separated to such a distance from one another that their mutual attractions become insensible* . They are therefore subjected to the undisturbed influence of repulsive forces. According to the theory of Laplace, caloric constitutes the sole agent of repulsion; and equal increments of temperature, being identical with equal increments of elasticity, are necessarily followed by equal expansions. "Sous une pression constante la densité d'un gaz étant, comme on l'a vu, réciproque à cette fonction de la température, son volume est proportionnel à cette fonction... la température est alors représentée par ce volume, et ses variations sont représentées par les variations du volume d'un gaz soumis à une pression constante."

It cannot be requisite to pursue this argument further, since it has been shown by Laplace, that both the law of Mariotte, and that of equal expansion discovered by Dalton and GayLussac, are mathematically derivable from the following suppositions:- that the molecules of gases are at such a distance that their mutual attractions are insensible;-that these molecules retain caloric by a principle of attraction;-that their mutual repulsion is due to the repulsion of the molecules of caloric;-and, finally, that this repulsion is only sensible at imperceptible distances. If these suppositions be conceded, the laws of Mariotte and of Dalton are susceptible of rigid demonstration, and are moreover applicable to all elastic fluids, whatever be the nature or the number of their molecules.

There is a third argument noticed by Dr. Prout, to which M. Dumas attaches much weight, in support of the doctrine of equality of atoms in a given volume. It is founded on the recent experiments of $\mathrm{MM}$. Delarive and Marcet, which show that all the gases, in equal volumes, have the same capacity

* Mécanique Céleste, livie xii. ch. i. tom. v. p. 89-91. 
for heat. Now it had long ago been suggested by Dr. Dalton as the most probable view of the relations of elastic fluids to heat, that "the quantity of heat belonging to the ultimate particles of all elastic fluids must be the same under the same pressure and temperature." Dulong and Petit have since inferred from their experiments, that the specific heats of several simple bodies in the solid state, when multiplied by their atomic weights, give a constant quantity as their product** This relation has more recently.been shown by M. Neumann to extend to several compound mineral substancest. Admitting, then, the equality of the specific heats of the gases when equal volumes are compared, and also that their ultimate atoms possess the same amount of heat, M. Dumas's conclusion, that equal volumes must contain the same number of ultimate atoms, is perfectly legitimate. But one of the elements of his calculation is erroneous $\neq$. M. Dulong, in his elaborate memoir on specific heat $\S$, has subsequently established the impossibility of obtaining, by the experimental process of Delarive and Marcet, even an approximative measure of the specific heats of the different gases, and has shown that the earlier results of Delaroche and Berard are still those most deserving of confidence. His own experiments, founded on the relations between the specific heats of gases and their powers of propagating sound, concur with those of Berard in indicating considerable differences in the specific heats of the gases, whether equal weights or bulks are made the objects of comparison. Substituting, then, these results for those of Delarive, we obtain, by the process of reasoning adopted by Dumas, the opposite conclusion, - that equal volumes of the different gases compared, contain unequal numbers of atoms $\|$.

* Ann. de Chimie et de Phys. tom. x. p. 405.

+ Poggendorff's Annalen, vol. xxiii. p. 32.

\pm Traité de Chimie appl. aux Arts, tom. i. p. 41.

$\$$ Ann. de Chimie et de Phys. tom. xli. p. 113.

If This argument is not urged as possessing more than a negalive force. The specific beats of the gases are not yet determined with certainty; and it is even doubtful whether, if obtained, they would faithfully represent the absolute heats, - a supposition manifestly involved in the principle of calculation, that the specific heats are equal to the absolute heat of one atom multiplied t,y the number of atoms in a given volume. It cannot, moreover, be denied, that the specific heats of hydrogen, oxygen and nitrogen, obtained by Delaroche, are so nearly the same in equal volumes, that, allowing for probable errors, they may safely be regarded as identical. Hence, upon the principle of Dulong and Petit, those three gases must contain the same number of atoms, and the weight of the atom of oxy. gen must be represented by 16 (the number adopted by Berzelius) instead of 8 . 
It has been the object of the foregoing remarks to prove that there does not exist, in the principles of general physics, any foundation for the new doctrine of Dr. Prout and M. Dumas, "that a given volume contains the same number of ultimate atoms in all the different gases*." These principles, however, with the exception of the relations of specific heats, though they do not furnish any support to such a doctrine, must be acknowledged to involve nothing that is contradictory to it. It must be considered, therefore, simply as an hypothesis, the value of which is to be estimated by its applicability to chemical phænomena. When tried by this test, it will be found wholly untenable, unless it be supported by a second and yet more improbable hypothesis, "the divisibility of the atom." Indeed, the single example of muriatic acid gas is sufficient to demonstrate its unsoundness. A volume of this gas is constituted of half a volume of hydrogen and half a volume of chlorine. The number of atoms in a volume of hydrogen is therefore double that in the same volume of muriatic acid gas. Nitrous gas, in like manner, must contain half the number of atoms that are contained in an equal volume of azote. The same is true of ammoniacal gas, of bydriodic acid gas, of hydrocyanic and chlorocyanic acid vapours, and of the vapour of sulphuret of carbon, when compared with an equal volume of one of their constituents. It may, then, be confidently asserted, that chemical phænomena, at least as they are now generally interpreted, are inconsistent with the notion of an equality of atoms in all gases, compound and simple.

It is solely upon this supposed numerical equality of atoms that Dr. Prout's second proposition is founded. Now if it has been shown that such equality is not derivable from physical principles, and is also inconsistent with known chemical facts, that proposition can be no longer maintained, except as an independent hypothesis; and we are compelled, by the rules of philosophizing, to recur to the simple and beautiful conception of the indivisibility of the atom, taught by the illustrious author of the atomic system. Several considerations may, moreover, be urged in favour of the doctrine of Dalton, that the mutually repulsive molecules of elastic fluids are identical

- It is not asserted that there do not exist any two gases which contain in the same volume the same number of ultimate atoms. On the contrary, most of the simple gases and vapours, and some of the compound gases, are generally believed to be thus similarly constituted. We object only to the raising what is true in certain individual examples into a general and necessary proposition. 
with the ultimate chemical atoms. We have already had occasion to refer to the postulates employed by Laplace as the basis of his profound mathematical inquiries into the constitution of elastic fluids. Now if heat be combined with the particles of matter by a principle of attraction or affinity (as supposed by Laplace), it is impossible to conceive such affinity to be exercised by aggregates of atoms, and yet not to be the attribute of the single atoms, of which such aggregates are composed. And if the ultimate atoms be endowed with an affinity for caloric, no reason can be assigned why union should not take place between caloric and each ultimate atom singly; nor, the molecules of heat being self-repulsive, why the ultimate atoms after such union should not become mutually repulsive. The contrary hypothesis of Dr. Prout involves the anomaly of supposing heat to have a combining affinity for two or more atoms, while it is destitute of such affinity for single atoms; and also that of supposing two atoms to have relations towards two atoms, or three towards three, which do not obtain between single atoms.

It is furthermore apparent, that the question respecting the mode of union between heat and the molecules of bodies, is not limited to the constitution of elastic fluids, but must equally comprehend the conditions of liquid and of solid. Now the relations of several simple bodies to heat, established by the experiments of Dulong and Petit, point unequivocally to the chemical atoms, as determining the measure of specific heat. In the thirteen simple substances which were the subjects of experiment, they found that the product of the specilic heats into the atomic weights was invariably a constant quantity, and consequently that the ultimate atoms contained precisely the same quantity of caloric. These results bave been since confirmed by various German experimenters*, and can only be reconciled with the doctrine of the combination of heat with the ultimate chemical atom.

In recapitulation, it has been shown,

1 st, That the law of Mariotte, and that of the equal expansibility of the different gases, are mathematically derived from elements altogether foreign to the numerical relations of their ultimate molecules; and that no corollary is contained in those laws, determining equality of atoms in a given volume of the different elastic fluids.

* See Mr. Johnston's excellent and comprehensive Report on Chemistry, in the Report of the First and Second Mectings of the British Association for the Advancement of Science, p. 418. 
2nd, That the most trustworthy experiments on the specific heats of the gases, combined with the law of Dalton, Dulong, and Neumann, lead to an opposite inference.

3rd, That several examples of chemical combination are inconsistent with the doctrine of numerical equalityof atoms in equal spaces of the different gases.

4 th, 'That the original hypothesis of Dalton, which contemplates the self-repulsive gaseous molecule as identical with the ultimate chemical atom, has in its favour the greatest amount of probabilities.

Manchester, June 7, 1834.

V. A Demonstration of the Parallelogram of Forces. By J. H. Pratr, Esq.*

I ET $P Q$ be two forces acting on a point, their directions $\mathcal{L}$ including an angle $\alpha$; and let $R$ be their resultant, acting in the same plane with $P$ and $Q$, and making an angle $\theta$ with $P$.

Suppose $\mathbf{P}$ to be equivalent to two forces $P_{1} P_{2}$, acting in direction of $R$, and perpendicular to this direction; and let $Q$ be equivalent to $Q_{1} Q_{2}$, acting similarly;

$$
\left.\therefore \begin{array}{l}
\mathbf{R}=\mathbf{P}_{1}+\mathbf{Q}_{1} \\
0=\mathrm{P}_{2}-\mathbf{Q}_{2}
\end{array}\right\}
$$

To find the relation between $P_{1}$ and $P$, we observe that since the law of resolution must be independent of the magnitudes of $P_{\text {and }} \mathrm{P}_{1}$ so long as their ratio is the same, $\mathrm{P}_{1}$ must be of the form $\quad \mathrm{P}_{\mathrm{L}}=\mathrm{P} \cdot f(\theta)$, where $f(\theta)$ is to be found.

$$
\text { Now, when } \begin{aligned}
\theta & =0_{1} \frac{\pi}{2}, \pi_{1} \frac{3 \pi}{2}, 2 \pi_{1} \ldots \\
P_{1} & =P, 0_{1}-P, 0_{1} P_{1} \quad \ldots
\end{aligned}
$$

and from these we see that if $n$ be any integer

$$
f\left(n \cdot \frac{\pi}{2}\right)=\cos \left(n \cdot \frac{\pi}{2}\right)
$$

When $\theta$ has any value not comprised in the above formula, the only conditions to be satisfied by $f(\theta)$ are, that the sum of the resolved parts of $P_{1}$ and $P_{2}$ in the direction of $P$ shall equal $P$, and perpendicular to this equal 0 :

$$
\therefore \quad \mathrm{P}=\mathrm{P}_{1} f(\theta)+\mathrm{P}_{2} f\left(\frac{\pi}{2}-\theta\right) \text {, }
$$

* Communicated by the Author. 